\title{
FACTORES DE RIESGOS PSICOSOCIALES EN AUXILIARES DE ENFERMERÍA DE UN HOSPITAL DE LA RED PÚBLICA EN LA CIUDAD DE BOGOTÁ, COLOMBIA
}

\section{PSYCHOSOCIAL RISK FACTORS IN PERSONAL ASSISTANT HOSPITAL NURSING CARE OF SECOND LEVEL IN THE CITY OF BOGOTA, COLOMBIA}

\author{
Cristian Arley Jimenez ${ }^{1}$, Margarita María Orozco ${ }^{2}$, Nelly Esther Caliz ${ }^{3}$
}

\begin{abstract}
${ }^{1}$ Enfermero. Universidad de Ciencias Aplicadas y Ambientales U.D.C.A, Bogotá, Colombia, e-mail: crjimenez@udca.edu. co; ${ }^{2}$ Enfermera, Especialista en Gerencia de la Salud Ocupacional, Magister en Prevención de Riesgos Laborales, Docente Programa de Enfermería, Facultad Ciencias de la Salud. Universidad de Ciencias Aplicadas y Ambientales U.D.C.A, Bogotá, Colombia, e-mail: morozco@udca.edu.co; ${ }^{3}$ Enfermera, Magister en psicología comunitaria, c.Dr. Salud Colectiva, Docente Programa de Enfermería, Facultad Ciencias de la Salud. Universidad de Ciencias Aplicadas y Ambientales U.D.C.A, Bogotá, Colombia, e-mail: ncaliz@udca.edu.co
\end{abstract}

Rev. U.D.C.A Act. \& Div. Cient. 20(1): 23-32, Enero-Junio, 2017

\section{RESUMEN}

El trabajo en enfermería es considerado como una actividad estresante, tendiente a fomentar la aparición del riesgo psicosocial, por las condiciones de trabajo, propias de los servicios hospitalarios y de la labor de enfermería, lo cual, puede desencadenar alteraciones o daños en la salud, de tipo físico, mental y emocional. El objetivo de esta investigación fue evaluar los factores de riesgos psicosociales en los auxiliares de enfermería, de un hospital de la red pública en la ciudad de Bogotá, Colombia. Estudio cuantitativo, de tipo descriptivo. La muestra estuvo compuesta por 90 auxiliares de enfermería; para la recolección de la información, se utilizó el cuestionario CoPsoQ-istas21, versión 2.0 2014, instrumento que evalúa la exposición al riesgo psicosocial, fundamentado en la prevención. Los resultados evidencian que el $84,5 \%$ de los participantes corresponden a sexo femenino, el 52,9\%, tiene una edad que no supera los 31 años y el 75,9\% tiene un contrato temporal. En cuanto a la exposición de riesgos psicosociales, las dimensiones más desfavorables para la salud fueron el ritmo de trabajo, 97,6\%; inseguridad sobre las condiciones de trabajo, 66,1\% y las exigencias de esconder emociones, $65,2 \%$. Respecto a las dimensiones favorables para la salud, se destaca el sentido de trabajo, $90,8 \%$ y las posibilidades de desarrollo, 90,7\%. La investigación evidencia las condiciones psicosociales que pueden repercutir en la salud de la población sujeto de estudio.

Palabras clave: Estrés, enfermería, factores de riesgo, condiciones de trabajo, Riesgo psicosocial.

\section{SUMMARY}

Nursing work is considered as a stressful activity, tending to promote the appearance of psychosocial risk, by the working conditions of the hospital services and nursing work, which can trigger alterations or damages in health, of type Physical, mental and emotional. The objective was to evaluate the psychosocial risk factors in the nursing auxiliaries of a public network hospital in the city of Bogotá, Colombia. Quantitative study, descriptive type. The sample consisted of 90 nursing auxiliaries; for the collection of the information, the questionnaire CoPsoQ-istas21, version 2.0 2014, instrument that evaluates the exposure to psychosocial risk, based on prevention, was used. The results show that $84,5 \%$ of the participants correspond to women, $52,9 \%$, have an age that does not exceed 31 years, and 75,9\% have a temporary contract. As for the exposure of psychosocial risks, the most unfavorable dimensions for health were the work rate $97,6 \%$, insecurity about working conditions $66,1 \%$ and the requirements to hide emotions $65,2 \%$; With respect to the dimensions favorable to health, it emphasizes the sense of work $90,8 \%$ and the possibilities of development $90,7 \%$. The research shows the psychosocial conditions that can affect the health of the population under study.

Key words: Stress, nursing, risk factors, working conditions, psychosocial risk. 


\section{INTRODUCCIÓN}

El trabajo es una actividad fundamental para la calidad de vida de las personas y le permite al ser humano satisfacer sus necesidades, mediante la producción de bienes y servicios, aunque se constituye en una paradoja, pues contiene elementos que, por un lado, pueden llevar a las personas a la excelencia, pero, por otro, puede producir alteraciones o daños a su salud, física, psicológica, mental y social (Moreno, 2011).

Estas alteraciones en la salud pueden estar determinadas por diferentes características de las condiciones laborales, como son: la organización del trabajo, la carga física y mental, la autonomía en la realización de las actividades y el contexto social del empleado (Moncada et al. 2014).

Dicha problemática puede ser resultado del cambio en los modelos laborales, sociales, culturales, económicos y del desarrollo tecnológico, que han originado nuevas técnicas de producción y tendencias de flexibilización laboral, lo que ha generado la incertidumbre, el trabajo precario, la inestabilidad en el empleo y una creciente presión para satisfacer las exigencias de la vida laboral moderna (Quiñones \& Rodríguez, 2015; ILO, 2010), cambios importantes, que plantean nuevos retos para la seguridad y la salud de los trabajadores, en la prevención del riesgo psicosocial (ILO, 2010).

Los factores psicosociales, como condiciones potenciales de producir daño, son situaciones presentes en el ámbito laboral, relacionadas con la organización del trabajo, el tipo de puesto, la realización de la tarea e, incluso, con el entorno, que afectan el desarrollo del trabajo y la salud de las personas trabajadoras y la probabilidad de que éstas condiciones generen en el trabajador una alteración en salud, es lo que se denomina riesgo psicosocial (Gil-Monte, 2012).

Los factores de riesgo son diversos en su naturaleza y origen y se constituyen en dimensiones interdependientes de un mismo constructo social y representan la realidad de la organización y las condiciones de trabajo, en las cuales, se encuentra inmerso el trabajador.

Se componen de seis grandes grupos: las exigencias psicológicas, el conflicto trabajo-familia, el control sobre el trabajo, el apoyo social y la calidad de liderazgo, las compensaciones en el trabajo y el capital social y, como dimensiones específicas, se encuentran, entre otras, el ritmo de trabajo, las exigencias cuantitativas, las exigencias emocionales, la doble presencia y el conflicto del rol (Moncada et al. 2014).

La falta de control de la exposición en cada uno de los grupos anteriormente descritos, puede producir resultados psicológicos, físicos y sociales negativos, como el estrés laboral, el agotamiento o la depresión (Gil-Monte, 2009; EU-OSHA, 2016). Para la Organización Internacional de Trabajo (OIT), el estrés es la respuesta física y emocional a un daño causado por un desequilibrio entre las exigencias percibidas, los recursos y las capacidades de un individuo, para hacer frente a esas exigencias (ILO, 2016).

El impacto en la salud de los elevados niveles de estrés varía de un individuo a otro y puede conllevar al deterioro de la salud, a partir de alteraciones físicas, como enfermedades gastrointestinales, cardiovasculares y musculo-esqueléticas, entre otras; de la misma forma, puede generar trastornos del comportamiento, como el deterioro de las relaciones interpersonales, la violencia, el abuso en el consumo de alcohol, tabaco y otras sustancias psicoactivas y trastornos mentales, como la ansiedad, la depresión e, incluso, suicidio. Todo lo anterior representa un enorme costo, en términos de sufrimiento humano y de carga económica, para la persona y la sociedad (Moreno \& Báez, 2010; ILO, 2016).

Los factores de riesgos psicosociales han venido en aumento, en la medida que se han modificado las condiciones laborales de la población. Al respecto, el panorama epidemiológico en Europa evidencia que el $25 \%$ de los trabajadores afirman experimentar estrés relacionado con el trabajo y un porcentaje similar declara que sus labores tienen un efecto negativo en su salud (EUROFOUND \& EU-OSHA, 2014).

En Colombia, según los resultados de la II Encuesta Nacional de Condiciones de Seguridad y Salud en el Trabajo (2013), se evidencia que los factores de riesgos psicosociales ocupan el segundo lugar de exposición, seguido de los de tipo ergonómico. Además, se observa el aumento del reporte de acoso laboral, con $9,4 \%$ y el incremento del $43 \%$, entre el 2009 y el 2012, del reconocimiento de los trastornos mentales de origen laboral, principalmente, los eventos, como ansiedad y depresión (Ministerio de Trabajo, 2013).

Numerosos estudios, a nivel mundial, indican el incremento a la exposición a riesgos psicosociales y al estrés, lo que evidencia el problema actual en el mundo laboral y ha motivado el interés, preocupación y desarrollo de investigaciones, por diversos científicos, de la conducta humana (Naranjo, 2009; Dos santos Trettene et al. 2016), quienes, a partir de modelos explicativos, buscan plantear respuestas a los efectos psicosociales de las condiciones de trabajo; el más destacado es el Modelo de demanda-control apoyo social de Karasek y Theorell (Gil-Monte, 2012).

El modelo demanda-control apoyo social establece, que las experiencias de estrés surgen cuando las demandas del trabajo son altas y, al mismo tiempo, la capacidad de control de la misma es baja; en este contexto, el riesgo psicosocial se origina de una situación ambiental o personal que influye 
sobre el individuo, planteándole demandas o exigencias que no controla o no puede atender, lo que representa una amenaza para la misma (Alcalde de Hoyos, 2010).

Las alteraciones en la salud, derivadas de los factores psicosociales y el estrés, influyen negativamente en el funcionamiento de las organizaciones, afectando su desempeño, debido a que se han relacionado como una de las causas asociadas al incremento de los accidentes de trabajo, el ausentismo laboral, la rotación de personal, la disminución del desempeño y la productividad, además los costos generados por esta problemática (Gómez et al. 2014).

El personal que trabaja en instituciones hospitalarias es considerado con un alto riesgo psicosocial; en esta categoría, se incluye el auxiliar de enfermería, quien tiene una función social importante, al proporcionar un cuidado integral, en la atención a individuos con diversas alteraciones en salud, desde el apoyo al diagnóstico médico, tratamiento y rehabilitación, hasta acciones propias de cuidado básico, bajo la responsabilidad y orientación de profesionales en salud (Ministerio de Salud y Protección Social, 2005).

Esta población, se encuentra expuesta a factores de riesgos psicosociales y es especialmente susceptible a desarrollar estrés y alteraciones en su salud física y mental, por cuanto el ejercicio de cuidar implica una exposición a diversos factores de riesgo, sobrecarga laboral y emocional, además de exigencias personales, ocupacionales y familiares (Cogollo \& Gómez, 2010; Basset et al. 2011). De igual manera, se han evidenciado otros factores, no menos importantes, como la deficiente retribución económica, la falta de liderazgo, previsibilidad, apoyo social y una mayor percepción de inseguridad laboral (Sora et al. 2011; Martínez et al. 2012), además de un alto riesgo de desarrollar Síndrome de Burnout (GilMonte, 2009; Avendaño et al. 2009; Gómez et al. 2009).

Lo expuesto anteriormente indica que el trabajo de enfermería en los servicios asistenciales, sin discriminar la formación técnica y profesional, se convierte en un riesgo para la salud, que se puede iniciar con síntomas físicos y cognitivo conductuales del estrés, pero que, a largo plazo, puede desencadenar alteraciones mayores en su salud física y mental (Moreno, 2011), las cuales, aumentan la prevalencia de ausentismo por enfermedad laboral en esta población, con respecto a otros miembros del equipo de salud (Bargas \& Monteiro, 2014).

Partiendo de los antecedentes y de la importancia de la exposición a los factores de riesgos psicosociales, surge una pregunta problema, c்uáles son los factores de riesgo psicosociales que pueden afectar la salud y el desempeño laboral de los auxiliares de enfermería que laboran en un hospital de la red pública de la ciudad de Bogotá?
El objetivo de la investigación fue identificar, valorar y analizar la exposición a los factores de riesgos psicosociales en los auxiliares de enfermería, de un hospital de la red pública, en Bogotá; se seleccionó esta población, por las altas demandas laborales que les caracteriza. La investigación permite aportar a la evidencia científica de estudios específicos para auxiliares de enfermería, a nivel nacional e internacional, además sirve de insumo para la planificación de las actividades preventivas en pro del bienestar laboral.

\section{MATERIALES Y MÉTODOS}

Tipo de estudio: La investigación se desarrolló bajo un enfoque cuantitativo, con diseño de un estudio descriptivo, de corte transversal.

Participantes: La población objeto de estudio para la evaluación del riesgo psicosocial, se conformó por auxiliares de enfermería, hombres y mujeres, mayores de 18 años, con diferentes modalidades de contratación y antigüedad laboral mayor a un mes, que labora en los servicios de hospitalización, unidad de cuidados intensivos, urgencias, salas de cirugía y sala de parto, de un hospital de la red pública en Bogotá, correspondiente a 134 auxiliares de enfermería.

Muestra: De tipo intencional, conformada por 90 participantes, quienes voluntariamente respondieron el cuestionario, equivalentes al $67 \%$ de la población. Como criterios de inclusión, se tuvieron en cuenta que fueran auxiliares de enfermería y laborar en áreas asistenciales. Se excluyeron aquellos que no respondieron por completo el cuestionario.

Variables de estudio: Se definieron variables sociodemográficas (edad, sexo), relativas a la contratación (antigüedad y relación laboral), variables relativas a las dimensiones psicosociales: ritmo de trabajo, inseguridad sobre las condiciones de trabajo, exigencias de esconder emociones, conflictos del rol, exigencias emocionales y exigencias cuantitativas.

Materiales e instrumentos: En el desarrollo de la investigación, se tuvo en cuenta los lineamientos establecidos en el CoPsoQ-istas21, versión 2.0 - 2014.

Para la recolección de la información, se utilizó el cuestionario CoPsoQ-istas21, versión 2.0- 2014, instrumento estandarizado, validado y fiable, de referencia en la disciplina de la psicología laboral, a nivel internacional, el cual, está basado en evidencia científica de la teoría general del estrés.

El cuestionario evalúo 5 grandes grupos y 20 dimensiones psicosociales, a las que se considera que un trabajador puede estar expuesto en el ámbito laboral; los valores de referencia de cada dimensión, se distribuyen en terciles, los cuales, han sido etiquetados de color verde, que incluyen, las pun- 
tuaciones más favorables para la salud: amarillo, puntuaciones intermedias y rojo, puntuaciones más desfavorables para la salud.

El instrumento contenía 109 preguntas cortas, con respuestas tipo Likert: 25 sobre las condiciones de empleo y trabajo, 69 relacionadas con la exposición a factores psicosociales y 15 sobre salud y bienestar personal. Fue aplicado previo consentimiento informado, en forma individual, anónima; el tiempo de diligenciamiento fue en promedio de 40 minutos y su recogida se realizó en un buzón, manteniendo el anonimato de la información. La sistematización de los datos obtenidos en las encuestas fue ingresada a una base de datos, para su procesamiento y análisis posterior. Para ello, se utilizó la aplicación informática que aporta el método CoPsoQistas21.

El análisis de la información, se realizó por medio de estadísticas descriptivas, que permitieron observar el comportamiento de las dimensiones estudiadas.

Consideraciones éticas: El estudio cumplió con los requerimientos establecidos por la Resolución 8430 de 1993, "por la cual se establecen las normas científicas, técnicas y administrativas para la investigación en salud" y fue clasificada como un estudio sin riesgo.

Tabla 1. Características de la población sujeto de estudio.

\section{RESULTADOS Y DISCUSIÓN}

La población participante del estudio fue auxiliares de enfermería, con un promedio de edad que no supera los 31 años, lo que significa que es una población joven, en etapa productiva. En cuanto al género, el $84,5 \%$ corresponde al femenino, condición socio-demográfica esperable en la población de enfermería, relacionada históricamente con el rol de cuidadoras, atribuido a la mujer. Al respecto, Mosqueda et al. (2013), lo señalan como una tendencia a la feminización del sector salud, desde el siglo XX hasta la fecha, lo que se debe tener en cuenta a la hora de analizar los factores psicosociales laborales, desde una perspectiva de género y que Dalri et al. (2014), ratifica en su investigación sobre carga horaria de trabajo de los enfermeros y su relación con las reacciones fisiológicas de estrés, en el que, el 89,4\% del personal de enfermería, es de sexo femenino.

Referente al tipo de contratación, el 75,9\% tuvo una relación laboral temporal, situación que responde a las actuales formas de contratación en el país, lo que no garantiza la perspectiva de un futuro digno como trabajador y mucho menos para sus familias (Morales, 2014). Esta condición, se agudiza con la variable antigüedad laboral, pues el $44 \%$ del personal ha trabajado entre 1 y 6 meses, aspecto que puede indicar una alta rotación de recurso humano, posiblemente, en busca de mejores condiciones laborales o por políticas internas de la empresa (Tabla 1).

\begin{tabular}{|c|c|c|c|c|}
\hline Características de la población & Variable & Escala de clasificación de la variable & $\mathbf{N}$ & $\%$ \\
\hline \multirow[t]{2}{*}{ Socio-demográficas } & Edad & $\begin{array}{l}\text { Menos de } 31 \text { años } \\
\text { Entre } 31 \text { y } 45 \text { años } \\
\text { Más de } 45 \text { años } \\
\text { No contesta }\end{array}$ & $\begin{array}{c}46 \\
30 \\
11 \\
3\end{array}$ & $\begin{array}{l}52,9 \\
34,5 \\
12,6\end{array}$ \\
\hline & Sexo & $\begin{array}{l}\text { Mujer } \\
\text { Hombre } \\
\text { No contesta }\end{array}$ & $\begin{array}{l}76 \\
13 \\
1\end{array}$ & $\begin{array}{l}85,4 \\
14,6\end{array}$ \\
\hline \multirow[b]{2}{*}{ Relativas a la contratación } & $\begin{array}{l}\text { Relación laboral con la } \\
\text { institución de trabajo }\end{array}$ & $\begin{array}{l}\text { Temporal } \\
\text { Planta } \\
\text { Operador } \\
\text { No contesta }\end{array}$ & $\begin{array}{l}63 \\
3 \\
4 \\
7\end{array}$ & $\begin{array}{l}75,9 \\
3,6 . \\
4,8\end{array}$ \\
\hline & Antigüedad laboral & $\begin{array}{l}\text { Menos de } 30 \text { días. } \\
\text { Entre } 1 \text { y hasta } 6 \text { meses. } \\
\text { Más de } 6 \text { meses y hasta } 2 \text { años. } \\
\text { Más de dos años. } \\
\text { No contesta. }\end{array}$ & $\begin{array}{l}7 \\
37 \\
20 \\
20 \\
6\end{array}$ & $\begin{array}{l}8,3 \\
44,0 \\
23,8 \\
23,8\end{array}$ \\
\hline
\end{tabular}


En cuanto a las dimensiones que constituyen los factores de riesgos psicosociales laborales en los auxiliares de enfermería, la investigación muestra las exposiciones al riesgo psicosocial por terciles, ordenadas en función de porcentajes y clasificadas, según el método, en: situación más desfavorable para la salud (rojo), intermedias o medianamente desfa- vorables para la salud (amarillas) y bajas o favorables para la salud (verdes).

Por lo amplio y las características del estudio, se presentarán las dimensiones más prevalentes, que pueden afectar o favorecer la salud de la población de auxiliares de enfermería (Tabla 2).

Tabla 2. Exposiciones al riesgo psicosocial del personal auxiliar de enfermería, ordenadas en función del porcentaje de trabajadores/as. En la situación más desfavorable para la salud (Rojo), intermedia (Amarilla) y favorables (verde).

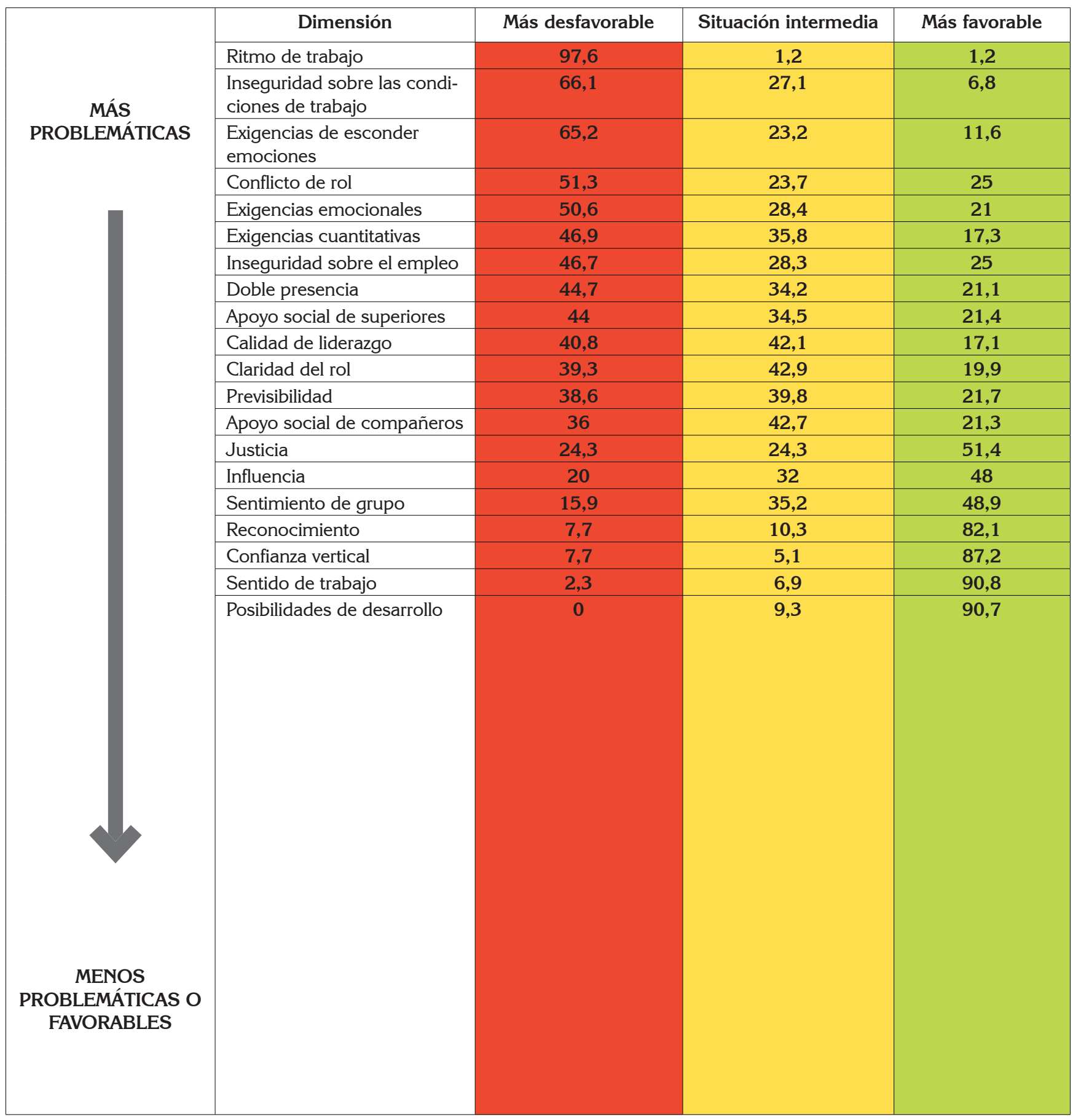


A continuación, un análisis descriptivo de los resultados más significativos de la investigación. En cuanto a las dimensiones desfavorables, los hallazgos fueron los siguientes:

Dimensión ritmo de trabajo: Esta dimensión corresponde a la exposición más desfavorable para la salud en la población sujeto de estudio, con un 97,6\% que, según Moncada et al. (2014), constituye la exigencia psicológica referida a la intensidad del trabajo. En este sentido, el estudio mostró que la población debe trabajar muy rápido y mantener un ritmo de trabajo alto durante toda la jornada, comportamiento significativamente superior al presentado por Ceballos et al. (2015), en el estudio sobre Factor psicosocial y carga mental en enfermería, en el cual, el $64 \%$ de los participantes manifestaron un volumen de trabajo elevado, en relación al tiempo disponible.

El alto ritmo de trabajo en auxiliares de enfermería responde a las condiciones laborales actuales a las que está sometido el trabajador de la salud, como las diversas formas de contratación, que han involucrado una mayor producción y prestación de servicios, con consecuencias, como: el aumento en las jornadas, la intensidad, el poco tiempo para desarrollar el trabajo y la falta de un reconocimiento salarial, acorde a la labor realizada (Mesa et al. 2010; Dalri et al. 2014; Hernández, 2015). También, el ritmo de trabajo se intensifica por otros factores, como servicios que demandan mayor atención de los pacientes por su complejidad, la falta de personal en las instituciones para cubrir las demandas y la presencia de dos vínculos de empleo (Barrios et al. 2012).

De acuerdo a lo anterior, el alto ritmo de trabajo y la sobrecarga laboral, traen consigo consecuencias, como los trastornos músculo-esqueléticos, el ausentismo o la disminución de la productividad (Moreno \& Báez, 2010; Trindade et al. 2014) y constituirse en un factor de riesgo psicosocial prioritario, que puede desencadenar alteraciones en la salud, como se evidenció en un estudio realizado por Sancinetti et al. (2009), en Sao Paulo, en Brasil, en el cual, los técnicos de enfermería fueron los que presentaron mayor cantidad de ausentismo, asociado a alteraciones osteomusculares, con un $41,5 \%$ y trastornos mentales y comportamentales, con un $28,4 \%$. En dicho estudio, el porcentaje mensual de licencias por enfermedad fue inversamente proporcional a la tasa de ocupación, sugiriendo que el personal de enfermería se ausentó por enfermedad, después de haber sido sometidos a un alto ritmo de trabajo.

Dimensión inseguridad sobre las condiciones de trabajo: Esta dimensión es entendida como la preocupación por el futuro, en cuanto a los cambios no deseados de aspectos laborales relacionados con el puesto, las tareas, el horario y el salario (Moncada et al. 2014) y que en la investigación de Torres et al. (2013), se asocia al gran compromiso que deben asumir en la institución y la incertidumbre que sienten cuando se acerca la fecha de terminación del contrato de trabajo.

Frente a esta dimensión, se encontró una exposición en el $66,1 \%$ de la población, resultado inferior al presentado por Bustillo et al. (2015), quienes evidenciaron que el $90 \%$ del personal que participó en su estudio indicó, como factor estresante, la falta de la estabilidad laboral y el $67,5 \%$, la rotación de turnos de trabajo, datos que contrastan con los resultados obtenidos por Acevedo et al. (2013), sobre sesgos psicosociales en el equipo de salud de Hospitales públicos en la Provincia de Córdoba, Argentina, en donde incluyen personal auxiliar de enfermería, donde solo el 19,3\% de los encuestados mencionó inseguridad y preocupación frente al contrato de trabajo.

La inseguridad frente a las condiciones de trabajo, es un nuevo factor de riesgo psicosocial, el cual, se explica, en razón a la situación económica actual y a los cambios sociales y políticos que han ocasionado nuevos modelos de contratación y han afectado al personal de la salud y, de manera importante el colectivo de la enfermería, es altamente estresante para esta población, quienes además de tener una alto ritmo y carga de trabajo, se tienen que enfrentar a cambios de turnos, rotaciones, salarios no acordes con la labor y la inseguridad sobre el tiempo de contratación (Sora et al. 2011).

Dimensión exigencias de esconder emociones: Son descritas como las demandas para mantener una apariencia neutral, independientemente del comportamiento de usuarios o clientes, compañeros, superiores u otras personas (Moncada et al. 2014). De acuerdo con los resultados obtenidos en la investigación, un $65,2 \%$ de los participantes, se encuentran expuestos a esta dimensión, porcentaje superior al referido por Acevedo et al. (2013), en donde el 49,6\% de los encuestados afirmó tener que guardar emociones y no expresarlas durante su trabajo.

En enfermería, las exigencias de esconder emociones, puede ser la respuesta a la carga emocional que se origina al brindar el cuidado, en un ambiente hospitalario impositivo, inflexible, normativo, competitivo y, en el cual, se pueden deteriorar las relaciones interpersonales, lo que origina la represión de sentimientos, emociones y la necesidad de mantener la imagen y el control ante sus pacientes, colegas y superiores (Seguel et al. 2015). Lo anterior, conlleva a la aparición de diversos sentimientos, aún más si se tiene en cuenta que estas exigencias se han convertido en un elemento esencial para la evaluación de la calidad del servicio prestado y la satisfacción del paciente, su familia y las instituciones (Moreno et al. 2010).

Dimensión conflicto del rol: Entendida, como aquellas exigencias contradictorias que se presentan en el trabajo y que 
pueden suponer conflictos de carácter profesional o ético (Moncada et al. 2014). La investigación evidenció una exposición desfavorable, en el 51,3\% de la población, comportamiento similar al de Campanario et al. (2011), quienes evidenciaron una exposición en el $50 \%$ de los encuestados, considerando, como situación estresante, realizar demasiadas tareas, que no son propias de su ejercicio profesional y comportamiento levemente inferior, al de Ceballos et al. (2015), quienes encontraron en el $47,7 \%$ de la población, que no había claridad del rol en su trabajo. Por el contrario, Acevedo et al. (2013) evidenciaron una exposición menos desfavorable, en un $29,5 \%$, en su estudio de riesgos psicosociales.

Al respecto del conflicto del rol, actualmente, las funciones que ejerce el personal auxiliar de enfermería, se caracterizan por el desarrollo de actividades propias y anexas, que exigen las instituciones en relación al cuidado de insumos, infraestructura, entre otros y tareas administrativas delegadas, que están generando el desplazamiento de las actividades propias del cuidado humano, que aumenta la carga de trabajo y conflictúan el que hacer del auxiliar (Mesa et al. 2010).

Dimensión exigencias emocionales: Se entiende, como las demandas para no dejarse involucrar en situaciones emocionales derivadas de las relaciones interpersonales, que implica el trabajo (Moncada et al. 2014). Al respecto, la investigación arrojó una exposición a esta dimensión, en el 50,6\% de los auxiliares de enfermería, comportamiento similar al estudio de Acevedo et al. (2013), quienes encontraron una exposición del 49,8\% y evidenciaron en la población, el desgaste emocional producido por el trabajo.

Las exigencias emocionales son factores de riesgos psicosociales específicos e intrínsecos al contenido de la tarea y a determinados eventos, íntimamente relacionados con la actividad que se realiza (De Puelles Casenave, 2009); en enfermería surgen en la interacción con el sujeto de cuidado, en la permanente confrontación de convivir con el sufrimiento, el dolor, la muerte; de igual forma, cuando el auxiliar se involucra en aspectos emocionales, familiares y sociales del paciente, lo que genera diversos sentimientos, como la preocupación, el miedo, el rechazo y la desesperación (Costa et al. 2009).

Al respecto, Martínez et al. (2012) plantean esta dimensión como un excelente predictor para el malestar mental, que puede generar sentimientos de ansiedad, desánimo y tristeza, síntomas conductuales, como irritabilidad, agobio, trastornos del sueño y síntomas cognitivos del estrés, entre ellos: dificultad para concentrarse, tomar decisiones, pensar de forma clara y falta de vitalidad.

Dimensión exigencias cuantitativas: Por sus características, el ritmo de trabajo, se encuentra estrechamente relacionado con la dimensión exigencias cuantitativas, definidas como las demandas psicológicas, derivadas de la cantidad de tareas, las cuales, son altas, cuando se tienen más actividades de las que se puede realizar, en el tiempo asignado (Moncada et al. 2014).

En la investigación, se identificó la exposición del 46,9\% de la población a esta dimensión; al respecto, el personal auxiliar de enfermería expresó no tener tiempo para realizar las tareas y la distribución de las mismas es irregular, comportamiento inferior al presentado por Bustillo et al. (2015), quienes encontraron que el $75,0 \%$ del personal de enfermería consideró que el tiempo disponible de trabajo no es suficiente para alcanzar a realizar todas sus tareas, al igual que Campanario et al. (2011), quienes evidenciaron que el $61,1 \%$ del personal auxiliar considera que un factor estresante es la falta de tiempo suficiente, para realizar todas sus labores.

Dicho hallazgo es contrario al encontrado por Acevedo et al. (2013), en su estudio dirigido a personal de la salud, donde se incluyen profesionales de enfermería y auxiliares, en el cual, el $67,8 \%$ de los encuestados mencionó poder hacer su trabajo con tranquilidad.

Esta dimensión, comúnmente, se encuentra relacionada, con la falta de personal, la incorrecta distribución de los tiempos, la inadecuada planificación del trabajo y, de igual manera, con otras condiciones, como el salario, la falta de herramientas, materiales o procesos de trabajo (Moncada et al. 2014).

Dimensiones favorables para la salud: Por lo amplio del estudio, se mencionarán las dimensiones más favorables o protectoras para la salud, las cuales, deben ser potenciadas en la población sujeto de estudio.

Los resultados fueron los siguientes: posibilidades de desarrollo, 90,7\%; sentido del trabajo, 90,8\%; confianza vertical, $87,2 \%$ y reconocimiento, $82,1 \%$. Al respecto, las posibilidades de desarrollo son consideradas como la posibilidad de que el auxiliar de enfermería ponga en práctica sus conocimientos y habilidades para aprender nuevas experiencias; el sentido de trabajo, como la oportunidad de poner en práctica los valores éticos, sentirse útil y darle un significado a las tareas que se realizan; la confianza vertical, como la seguridad de que la dirección y los trabajadores actuarán competentemente y el reconocimiento, como la valoración, el respeto y el trato justo por parte de la dirección en el trabajo (Moncada et al. 2014).

Finalmente, se concluye que el trabajo del auxiliar de enfermería en los servicios asistenciales, es de alto riesgo psicosocial, lo que puede conllevar a corto, mediano y largo plazo alteraciones y daños en la salud física, mental y emocional, 
además de repercutir, a nivel organizacional, por los altos costos derivados del ausentismo, disminución de la productividad y la calidad del servicio.

Por lo anterior, el estudio confirma la importancia de documentar, identificar, valorar y controlar los factores de riesgo psicosociales en la población de auxiliares de enfermería, así como la necesidad de generar estrategias de prevención y control de estos factores de riesgo, con el fin de prevenir alteraciones en la salud, mejorar sus condiciones de trabajo y bienestar y, de esta manera, se fomentan ambientes laborales más saludables, se mejora la productividad, la calidad del servicio y el cuidado humanizado, esencial en la atención de enfermería.

Agradecimientos: Los autores agradecen al Docente Luis David Olaya, por la colaboración prestada en la investigación. Financiación: El estudio fue financiado por la Universidad de Ciencias Aplicadas y Ambientales U.D.C.A. Conflicto de intereses: El manuscrito fue preparado y revisado con la participación de los autores, quienes declaramos que no existe conflicto de intereses que ponga en riesgo la validez de los resultados presentados.

\section{BIBLIOGRAFÍA}

1. ACEVEDO, G.; SÁNCHEZ, J.; FARÍAS, M.; FERNÁNDEZ, A. 2013. Riesgos Psicosociales en el Equipo de Salud de Hospitales Públicos de la Provincia de Córdoba, Argentina. Ciencia \& Trabajo. (Chile). 15(48):140147.

2. ALCALDE DE HOYOS, J. 2010. Estrés laboral. Informe técnico sobre estrés en el lugar de trabajo. Disponible desde Internet en: http://www.juntadeandalucia. es/salud/export/sites/csalud/galerias/documentos/ c_3_c_1vida_sana/promocion_salud_lugar_trabajo/estres_laboral_informe_pslt.pdf (con acceso 23/02/2016).

3. AVENDAÑO, C.; BUSTOS, P.; ESPINOZA, P.; GARCÍA, F.; PIERART, T. 2009. Burnout y apoyo social en personal del servicio de psiquiatría de un hospital público. Ciencia y enfermería. (Chile).15(2):55-68.

4. BARGAS, E.; MONTEIRO, M. 2014. Fatores relacionados ao absenteísmo por doença entre trabalhadores de Enfermagem. Acta Paulista de Enfermagem. (Brasil). 27(6):533-538.

5. BARRIOS, A.; ARECHABALA, M.; VALENZUELA, V. 2012. Relación entre carga laboral y burnout en enfermeras de unidades de diálisis. Enfermería Nefrológica. (España). 15(1):46-55.
6. BASSET, M.; ESTÉVEZ, R.; LEAL, G.; GRANADOS, G.; LÓPEZ, V. 2011. Estrés laboral y personal en los recursos humanos de Enfermería de una Unidad de Psiquiatría en México, D.F. Enf Neurol. 10(1):27-31.

7. BUSTILLO, M.; ROJAS, J.; SÁNCHEZ, A.; SÁNCHEZ, L.; MONTALVO, A.; ROJAS, M. 2015. Riesgo psicosocial en el personal de enfermería. Servicio de urgencias en hospital universitario de Cartagena. Duazary. (Colombia). 12(1):32-40.

8. CAMPANARIO, R.; REY, M.; ORTEGA, A.; CARDENO, M. 2011. Situaciones estresantes para el personal de enfermería en observación del hospital universitario virgen del rocío. Revista científica de la Sociedad española de enfermería de urgencias. (España). 2(17):1-4.

9. CEBALlOS, P.; ROLO, G.; HERNÁNDEZ, E.; DÍAZ, D.; PARAVIC, T.; BURGOS, M. 2015. Psychosocial factors and mental work load: a reality perceived by nurses in intensive care units. Rev. Latino-am. Enfermagem. (Brasil). 23(2):315-322.

10. COGOLLO, M.; GOMEZ, B. 2010. Condiciones laborales en enfermeras de Cartagena, Colombia. Av. Enferm. 28(1):31-38.

11. COSTA, F.; VIEIRA, M.; SENA, ROSENI, R. 2009. Absenteísmo relacionado à doenças entre membros da equipe de enfermagem de um hospital escola. Rev. Bras. Enfermagem. 62(1):38-44.

12. DALRI, R.; SILVA, L.; MENDES, A.; ROBAZZI, M. 2014. Carga horaria de trabajo de los enfermeros y su relación con las reacciones fisiológicas de estrés. Rev. Latino-Am. Enfermagem. 22(6):959-965.

13. DE PUELLES CASENAVE, C. 2009. Exigencia y carga emocional del trabajo policial: la intervención policial ante catástrofes y emergencias masivas. Psicopatología clínica legal y forense. (España). 9:171-196.

14. DOS SANTOS TRETTENE, A.; SOARES DE ANDRADE, C.; VON KOSTRISCH, L.; MERIGHI, M.; RIBEIRO, A. 2016. stress in nursing professionals working at a specialized hospital. Rev. Enfermagem. (Brasil). 10(12):4450-4458.

15. EUROFOUND; EU-OSHA. 2014. Psychosocial risks in Europe: Prevalence and strategies for prevention, Publications Office of the European Union, Luxembourg. Disponible desde Internet en: file:///C:/Users/ internet_tt/Downloads/Report\%20co-branded\%20 
EUROFOUND\%20and\%20EU-OSHA.pdf (con acceso $13 / 04 / 2016)$.

16. EUROPEAN AGENCY FOR SAFETY HEALTH AT WORK -EU-OSHA-. 2016. Psychosocial risks and stress at work. Disponible desde Internet en: https://osha.europa. $\mathrm{eu} / \mathrm{en} /$ themes/psychosocial-risks-and-stress (con acceso 16/11/2016).

17. GIL-MONTE, P. 2012. Riesgos psicosociales en el trabajo y salud ocupacional. Rev. Perú. Med. Exp. Salud pública. 29(2):237-241.

18. GIL-MONTE, P. 2009. Algunas razones para considerar los riesgos psicosociales en el trabajo y sus consecuencias en la salud pública. Rev. Española de Salud Pública. 83(2):169-173.

19. GÓMEZ, P.; HERNÁNDEZ, J.; MÉNDEZ, M. 2014. Factores de riesgo psicosocial y satisfacción laboral en una empresa chilena del área de la minería. Ciencia \& Trabajo. 16(49):9-16.

20. GÓMEZ, M.; ÁLAMO, M.; AMADOR, M.; CEACERO, F.; MAYOR, A.; MUÑOZ GONZÁLEZ, A.; IZQUIERDO, M. 2009. Estudio de seguimiento del desgaste profesional en relación con factores organizativos en el personal de enfermería de medicina interna. Med. Segur. Trab. (España). 55(215):52-62.

21. HERNÁNDEZ, C. 2015. Flexibilidad laboral: cifin del trabajo permanente? Rev de Estudios Interdisciplinarios en Ciencias Sociales. Telos. (Venezuela). 18(2):250265.

22. INTERNATIONAL LABOUR ORGANIZATION -ILO- . 2010. Emerging risks and new patterns of prevention in a changing world of work. Disponible desde Internet en: http://www.ilo.org/public/portugue/region/ eurpro/lisbon/pdf/28abril_10_en.pdf (con acceso 16/11/2016).

23. INTERNATIONAL LABOUR ORGANIZATION -ILO-. 2016. Workplace stress: a collective challenge. Disponible desde Internet en: http://www.ilo.org/wcmsp5/ groups/public/---ed_protect/---protrav/---safework/ documents/publication/wcms_473267.pdf (con acceso 16/11/2016).

24. MARTÍNEZ, M.; ALBORES, L.; MÁRQUEZ, M. 2012. La integridad mental del personal clínico de un hospital psiquiátrico, asociada a una alta exigencia emocional y a la organización nociva del trabajo. Salud Mental. (México). 35(4):297-304.
25. MESA, L.; ROMERO, B.; MARÍA, N. 2010. Profesionales de enfermería y cuidado en las condiciones laborales actuales. Invest. Enferm.: Imagen y Desarrollo. (Colombia). 12(2):55-92.

26. MINISTERIO DE SALUD. 1993. Resolución Na 8430. Por la cual se establecen las normas científicas, técnicas y administrativas para la Investigación en salud. Disponible desde Internet en: https://www.minsalud. gov.co/Normatividad Nuevo/RESOLUCION\%20 8430\%20DE\%201993. pdf (con acceso 15/092016).

27. MINISTERIO DE SALUD Y PROTECCION SOCIAL. 2005. Perfiles ocupacionales y normas de competencia laboral para auxiliares en las áreas de la salud. Disponible desde Internet en: https://www.minsalud. gov.co/salud/Documents/Perfiles\%20Ocupacionales.pdf (con acceso 28/02/2017).

28. MINISTERIO DE TRABAJO. 2013. Informe Ejecutivo de la Segunda Encuesta Nacional de Condiciones de Seguridad y Salud en el Trabajo en el Sistema General de Riesgos Laborales de Colombia. Disponible desde Internet en: http://www.fasecolda.com/ index.php/ramos/riesgos-laborales/documentos-deinteres/ (con acceso 10/11/2016).

29. MONCADA, S.; LLORENS, C.; ANDRÉS, R.; MORENO, N.; MOLINERO, E. 2014. Manual del método CoPsoQ-istas21 (versión 2) para la evaluación y la prevención de los riesgos psicosociales en empresas con 25 o más trabajadores y trabajadoras versión media. Disponible desde Internet en: http:// www.istas.net/copsoq/ficheros/documentos/v2/manual\%20Copsoq\%202(24-07-2014).pdf (con acceso 11/03/2016).

30. MORALES, E. 2014. La enfermería una fuerza para el cambio: recurso vital para la salud. Rev. ANEC. (Colombia) 77:2-3.

31. MORENO, B.; GÁLVEZ, M.; RODRÍGUEZ, R.; GARROSA, E. 2010. Emociones y salud en el trabajo: análisis del constructo "trabajo emocional" y propuesta de evaluación. Rev. Latinoam. Psicología. (Colombia). 42(1): 63-73.

32. MORENO, B.; BAEZ, C. 2010. Factores y riesgos psicosociales, formas, consecuencias, medidas y buenas prácticas. Disponible desde Internet en: http://www. insht.es/InshtWeb/Contenidos/Documentacion/PUBLICACIONES\%20PROFESIONALES/factores\%20 riesgos\%20psico.pdf (con acceso 15/092016). 
33. MORENO, B. 2011. Factores y riesgos laborales psicosociales: conceptualización, historia y cambios actuales. Medicina y seguridad del trabajo. (España). 57(1):4-19. 38.

34. MOSQUEDA, A.; PARAVIC, T.; VALENZUELA, S. 2013. División sexual del trabajo y Enfermería. Index de Enfermería. (España). 22(1):70-74. 39.

35. NARANJO, M. 2009. Una revisión teórica sobre el estrés y algunos aspectos relevantes de éste en el ámbito educativo. Rev. Educación. (Costa Rica). 33(2):171190.

36. QUIÑONES, C.; RODRÍGUEZ, S. 2015. La reforma laboral, la precarización del trabajo y el principio de estabilidad en el empleo. Rev. Latinoam. Derecho Social. (México). 21:179-201.

37. SANCINETTI, T.; GAIDZINSKI, R.; ANDRES, V.; TOGEIRO, F.; CAMPOS, P.; CIAMPONE, M.; KURCGANT, P.; DA SILVA, F. 2009. Absenteeism - disease in the nursing staff: relationship with the occupation tax. Rev. Escola Enfermagem da USP. (Brasil). 43(2):1277 1283.
38. SEGUEL, F.; VALENZUELA, S.; SANHUEZA, O. 2015. El trabajo del profesional de enfermería: revisión de la literatura. Ciencia y Enfermería XXI. (Chile). 21(2):1120.

39. SORA, B.; CABALLER, A.; PEIRÓ, J. 2011. Consecuencias de la inseguridad laboral. El papel modulador del apoyo organizacional desde una perspectiva multinivel. Psicothema. (España). 23(3):394-400.

40. TRINDADE, L; GRISA, C.; OSTROVSKI, V.; ADAMY, E.; FERRAZ, L; AMESTOY, S.; COELHO, A.; BORDIGNON, M. 2014. Absenteísmo na equipe de enfermagem no ambiente hospitalar. Enfermería Global. (Brasil). 13(36):138-146.

41. TORRES, A.; ACOSTA, M.; CRUZ, Á. 2013. Burnout y estrategias de afrontamiento en personal de atención en salud en una empresa social del estado nivel 2. Rev. Col. Salud Ocupacional. 3(4):16-19.

Recibido: Julio 10 de 2016

Aceptado: Mayo 17 de 2017

Cómo citar:

Jiménez, C.A.; Orozco, M.M.; Caliz, N.E. 2017. Factores de riesgos psicosociales en auxiliares de enfermería de un hospital de la red pública en la ciudad de Bogotá, Colombia. Rev. U.D.C.A Act. \& Div. Cient. 20(1): 23-32. 